\title{
Stellar mass ejections
}

\author{
Moira Jardine ${ }^{1}$, Jean-Francois Donati ${ }^{2}$ and Scott G. Gregory ${ }^{3}$ \\ ${ }^{1}$ SUPA, School of Physics and Astronomy, University of St Andrews, North Haugh, \\ St Andrews, KY16 9SS, UK \\ email: $\mathrm{mmj}$ @st-andrews.ac.uk \\ ${ }^{2}$ LATT, CNRS-UMR 5572, Obs. Midi-Pyrénées, 14 Av. E. Belin, F-31400 Toulouse, France \\ email: donati@ast.obs-mip.fr \\ ${ }^{3}$ SUPA, School of Physics and Astronomy, University of St Andrews, North Haugh, \\ St Andrews, KY16 9SS, UK \\ email: sg64@st-andrews.ac.uk
}

\begin{abstract}
It has been known for some time now that rapidly-rotating solar-like stars possess the stellar equivalent of solar prominences. These may be three orders of magnitude more massive than their solar counterparts, and their ejection from the star may form a significant contribution to the loss of angular momentum and mass in the stellar wind. In addition, their number and distribution provide valuable clues as to the structure of the stellar corona and hence to the nature of magnetic activity in other stars.

Until recently, these "slingshot prominences" had only been observed in mature stars, but their recent detection in an extremely young star suggests that they may be more widespread than previously thought. In this review we will summarise our current understanding of these stellar prominences, their ejection from their stars and their role in elucidating the (sometimes very non-solar) behaviour of stellar magnetic fields.
\end{abstract}

Keywords. stars:magnetic fields, stars:coronae, stars:imaging, stars:spots

\section{Introduction}

During this Symposium we have learned a great deal about the Sun and its influence on its environment, but in this review we want to begin by addressing the question "How typical is the Sun as a star?" Stars on the main sequence (i.e. those stars that have settled into the longest phase of their lives, when they are burning hydrogen in their cores) can have very different interior structures depending on their mass, yet magnetic activity is almost ubiquitous among them. High mass stars have a convectively stable (or radiative) outer envelope, so how do they generate their magnetic fields? They can do this in their convective cores, although this raises the question of how to transport the flux to the surface (Charbonneau \& MacGregor 2001; Brun et al. 2005). They can also generate magnetic fields in the radiative zone, but a very non-solar dynamo process (Spruit 2002; Tout \& Pringle 1995; MacDonald \& Mullan 2004; Mullan \& MacDonald 2005; Maeder \& Meynet 2005). Alternatively, the fields may be fossils, left over from the early stages of the formation of the star (Moss 2001; Braithwaite \& Spruit 2004; Braithwaite \& Nordlund 2006). Very low mass stars also have an internal structure that is very different from that of the Sun in that convection may extend throughout their interiors. In the absence of a tachocline, these stars cannot support a solar-like interface dynamo, yet they, like the high mass stars, exhibit observable magnetic fields. The mechanism by which they generate these magnetic fields has received a great deal of attention recently. While a decade or so ago, it was believed that these stars could only generate small-scale magnetic fields (Durney et al. 1993; Cattaneoe 1999), more recent studies have suggested that large 


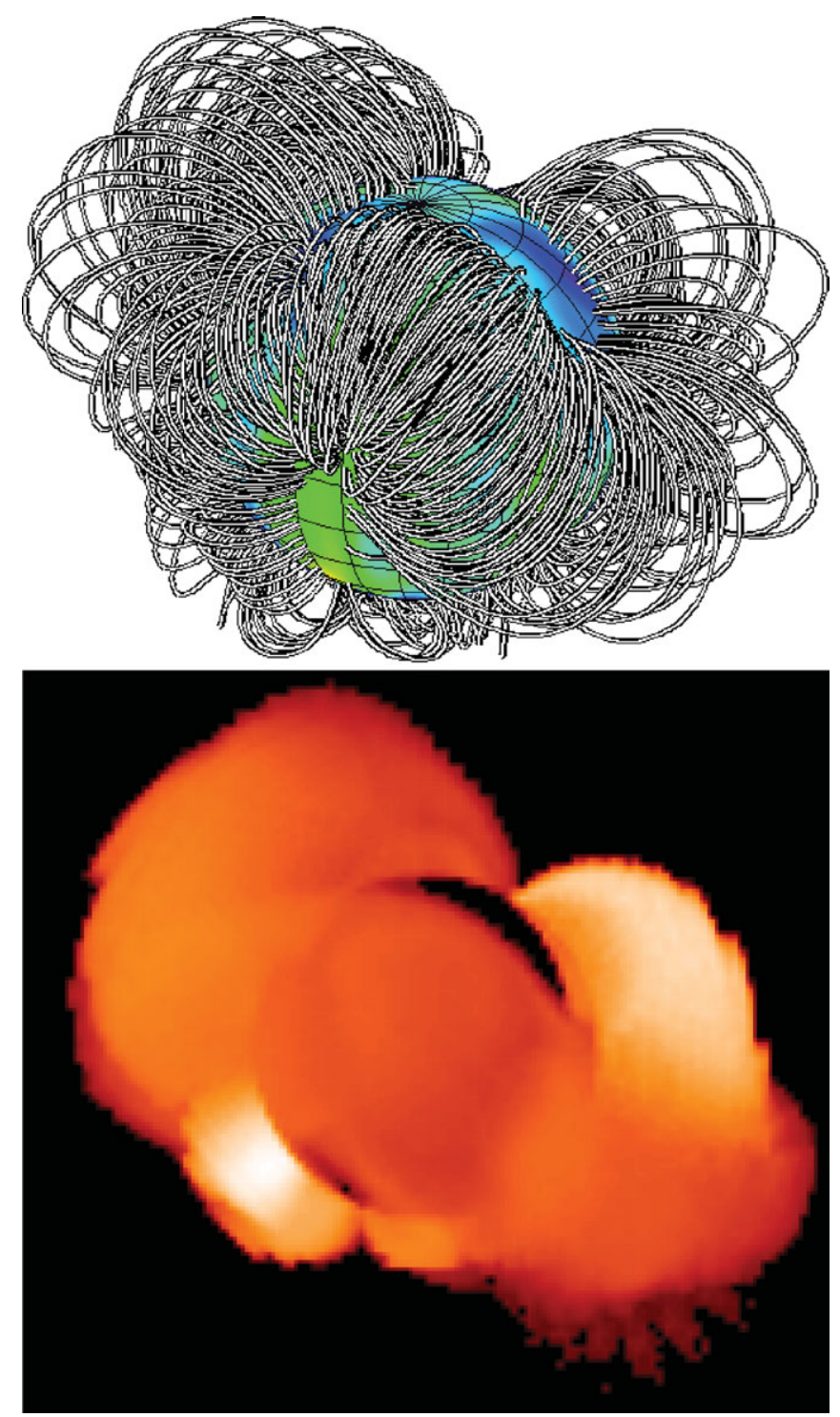

Figure 1. Closed field lines (top) and corresponding X-ray image (bottom) for the rapidly-rotating star LQ Hya. A coronal temperature of $10^{6} \mathrm{~K}$ is assumed.

scale fields may be generated. These models differ, however, in their predictions for the form of this field and the associated latitudinal differential rotation. They predict that the fields should be either axisymmetric with pronounced differential rotation (Dobler et al. 2006), non-axisymmetric with minimal differential rotation (Küker \& Rüdiger 1997, 1999; Chabrier \& Küker 2006) or, in a very recent model, axisymmetric with negligible differential rotation (Browning 2008).

\section{Observing stellar prominences and magnetic fields}

With this bewildering array of magnetic field geometries, the nature of any prominences that might be confined in and ultimately ejected from these coronae becomes even more 

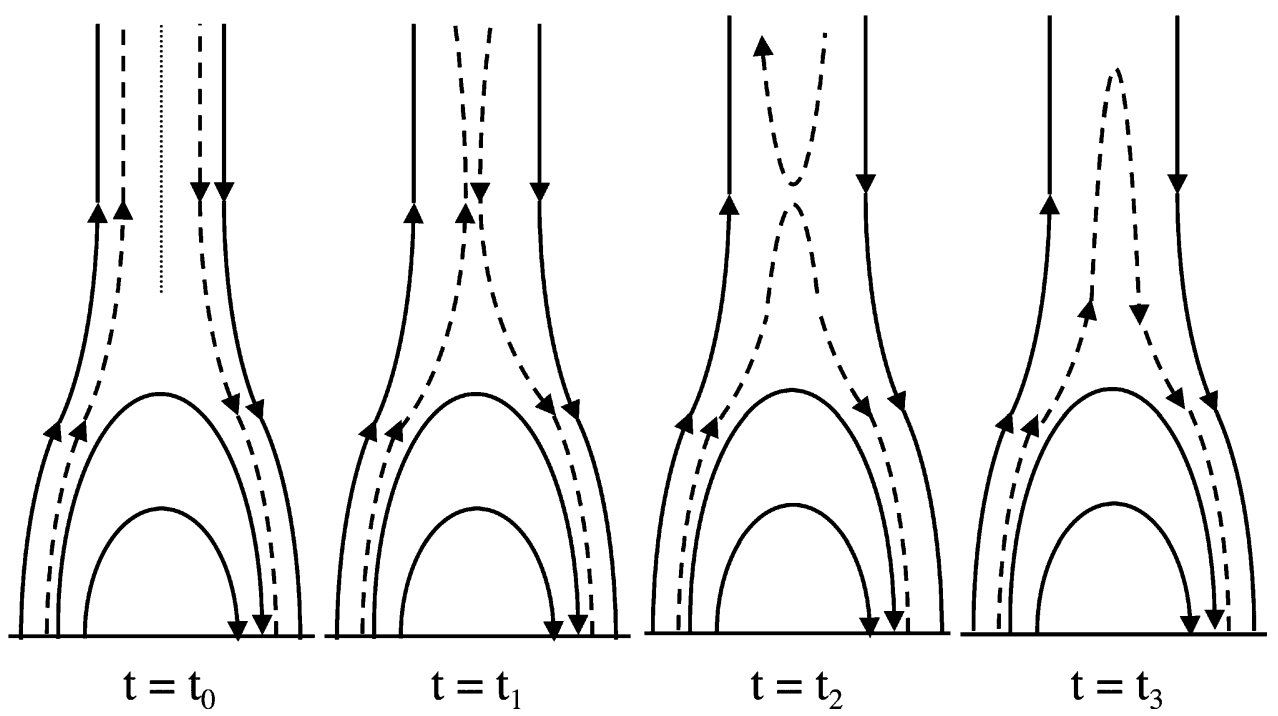

Figure 2. A schematic diagram of the formation of prominence-bearing loops. Initially, at $t=t_{0}$ a current sheet is present above the cusp of a helmet streamer. Reconnection in the current sheet at $t=t_{1}$ produces a closed loop at $t=t_{2}$. The stellar wind continues to flow until pressure balance is restored, thus increasing the density in the top of this new loop. Increased radiative losses cause the loop to cool and the change in internal pressure forces it to a new equilibrium at $t=t_{3}$.

interesting, but detecting their presence is not an easy task. They can, however, be observed in rapidly-rotating stars as transient $\mathrm{H} \alpha$ absorption features (Collier Cameron \& Robinson 1989b,a; Collier Cameron \& Woods 1992; Jeffries 1993; Byrne et al. 1996; Eibe 1998; Barnes et al. 2000; Donati et al. 2000). In many instances these features re-appear on subsequent stellar rotations, often with some change in the time taken to travel through the line profile. These features are interpreted as arising from the presence of clouds of cool, dense gas co-rotating with the star and confined within its outer atmosphere. As many as six may be present in the observable hemisphere. What is most surprising about them is their location, which is inferred from the time taken for the absorption features to travel through the line profile. Values of several stellar radii from the stellar rotation axis are typically found, suggesting that the confinement of these clouds is enforced out to very large distances. Indeed the preferred location of these prominences appears to be at or beyond the equatorial stellar co-rotation radius, where the inward pull of gravity is exactly balanced by the outward pull of centrifugal forces. Beyond this point, the effective gravity (including the centrifugal acceleration) points outwards and the presence of a restraining force, such as the tension in a closed magnetic loop, is required to hold the prominence in place against centrifugal ejection. The presence of these prominences therefore immediately requires that the star have many closed loop systems that extend out for many stellar radii. Maps of the surface brightness distributions of these stars can be obtained by Doppler imaging, while magnetograms are now almost routinely possible with Zeeman-Doppler imaging. These maps typically show a complex distribution of surface spots that is often very different from that of the Sun, with spots and mixed polarity flux elements extending over all latitudes up to the pole (Donati \& Collier Cameron 1997; Donati et al. 1999; Strassmeier 1996).

From these magnetograms we can extrapolate the coronal magnetic field using a $\mathrm{Po}$ tential Field Source Surface method (Altschuler \& Newkirk, Jr. 1969; Jardine et al. 1999, 


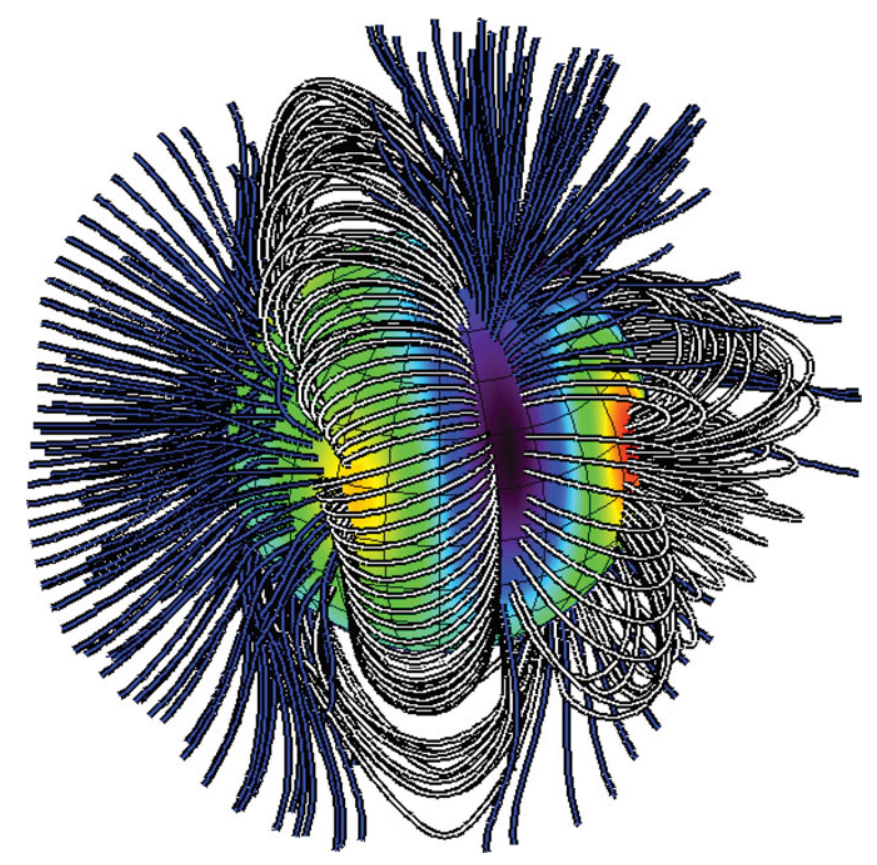

Figure 3. Closed field lines (white) and open field lines (blue) extrapolated from a Zeeman-Doppler image of Tau Sco.

2001, 2002a; McIvor et al. 2003), or using non-potential fields (Donati 2001; Hussain et al. 2002). By assuming that the gas trapped on these field lines is in isothermal, hydrostatic equilibrium, we can determine the coronal gas pressure, subject to an assumption for the gas pressure at the base of the corona. We assume that it is proportional to the magnetic pressure, i.e. $p_{0} \propto B_{0}^{2}$, where the constant of proportionality is determined by comparison with X-ray emission measures (Jardine et al. 2002b, 2006; Gregory et al. 2006a). For an optically thin coronal plasma, this then allows us to produce images of the X-ray emission, as shown in Fig. 1. This immediately highlights one of the greatest puzzles of stellar prominences: that they are confined to a such great distances - several stellar radii - that they may well be outside the extent of the closed, X-ray emitting corona.

One way out of this problem is to confine the prominences in the wind region beyond the closed corona. Jardine \& van Ballegooijen (2005) have produced a model for this that predicts a maximum height $y_{m}$ for the prominence as a function of the co-rotation radius, $y_{K}$ where

$$
\begin{aligned}
\frac{y_{m}}{R_{\star}} & =\frac{1}{2}\left(-3+\sqrt{1+\frac{8 G M_{\star}}{R_{\star}^{3} \omega^{2}}}\right) \\
& =\frac{1}{2}\left(-3+\sqrt{1+8\left[\frac{y_{K}}{R_{\star}}+1\right]^{3}}\right) .
\end{aligned}
$$

Fig. 2 shows the sequence of events that might lead to the formation of one of these "slingshot" prominences. The stellar wind flows along the open field lines that bound a closed field region, forming a helmet streamer. If the current sheet that forms between these oppositely-directed field lines reconnects, then a loop of magnetic field will be formed. The stellar wind will continue to flow for a short time, until pressure balance is 


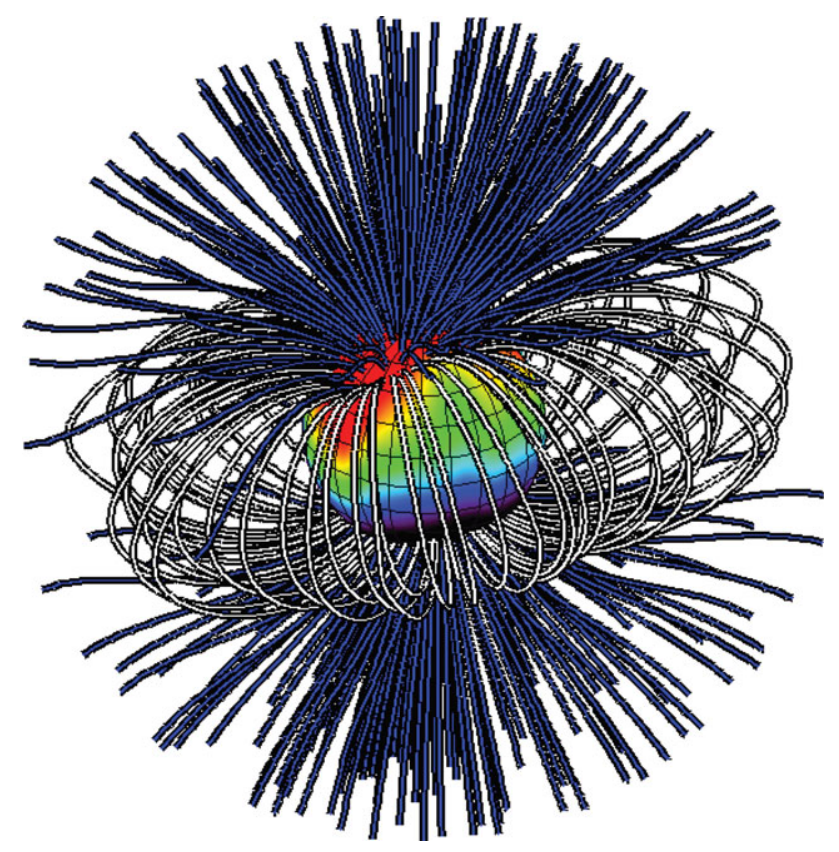

Figure 4. Closed field lines (white) and open field lines (blue) extrapolated from a Zeeman-Doppler image of V374 Peg.

re-established with a new field configuration. Jardine \& van Ballegooijen (2006) showed that a new, cool equilibrium was possible which could reach out well beyond the corotation radius. The distribution of prominence heights shown in Dunstone et al. (2008a,b) for the ultra-fast rotator Speedy Mic shows prominences forming up to (but not significantly beyond) this maximum height.

\section{Stellar magnetic field variation with stellar mass and evolutionary state}

But what of the many stars whose internal structure is very different from that of the Sun? Surface magnetograms are now available for stars of a range of masses. At the high mass end, Tau Sco is a very interesting example. At $15 \mathrm{M}_{\odot}$ it has a radiative interior, and yet as shown in Fig. 3 it displays a complex, strong field (Donati et al. 2006b). If this is a fossil field, it might be expected to be a simple dipole, but the very youth of this star, at only a million years, may be the reason why the higher-order field components have not yet decayed away. Interestingly, Tau Sco shows $\mathrm{H}_{\alpha}$ absorption features that are very similar to prominence signatures in lower mass stars. In this case, however, they are attributed to a "wind-compressed disk" that forms when sections of the very massive wind emanating from different parts of the stellar disk collide and cool (Townsend \& Owoki 2005). In contrast, as shown in Fig. 4 the very low mass fully-convective star V374 Peg has a very simple, dipolar field (Donati et al. 2006a). The highly-symmetric nature of the field and the absence of a measureable differential rotation are consistent with the recent models of Browning (2008). It is unfortunately not possible at present to detect any prominences that might be present on these very low mass stars because they stars are intrinsically too faint. Their detection would, however, be a very clear test of the magnetic structure, since in a simple dipole any prominences should, by symmetry, form in the equatorial plane. 


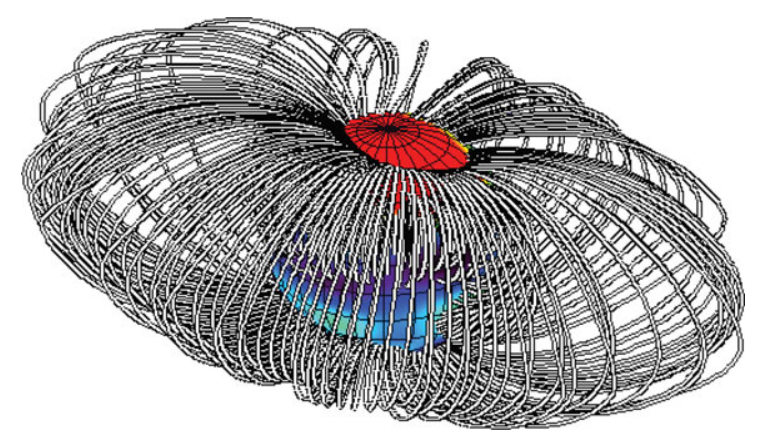

Figure 5. Closed field lines structure extrapolated from Zeeman-Doppler image of BP Tau.

It appears that stars with different internal structures may have dynamos that produce very different types of magnetic field. In particular, the transition from a solar-type interior to one in which the convective zone extends throughout the star appears to be associated with a decrease in field complexity. This transition happens for solar mass stars as they evolve from their very earliest stages when they are fully-convective, through the development of a radiative core as they approach the main sequence. Any associated change in the magnetic field structure is potentially very important, since the magnetic field is believed to channel the flow of material from the accretion disk that surrounds such young stars onto hotspots on the stellar surface. Significant advances have been made in the study of this magnetospheric accretion recently, with the advent of large-scale 3D MHD codes. It appears that the structure of the magnetic field can be a crucial factor in determining the nature of the accretion (Rekowski \& Brandenburg 2004; Gregory et al. 2006b; Long et al. 2007).

It is not only the flow of material onto the star that is important, however. The loss of both mass and angular momentum in a wind is also a crucial issue since these young stars should spin up as they contract, but they are observed to have typically only moderate rotation rates. This spin-down may be achieved through the exchange of magnetic torques between the star and the disk (known as disk-locking) or through a wind (Königl 1991; Collier Cameron \& Campbell 1993; Shu et al. 1994; Matt \& Pudritz 2005).

Determining the structure of the coronal magnetic field of these young stars is a difficult problem, however, since there are many factors than can influence it. As the stellar magnetic field drags through the disk it will be sheared and may be opened up entirely (Lynden-Bell \& Boily 1994). This shearing may act to deposit energy in the corona through reconnection between the magnetic fields of the star and the disk - certainly, some of the very large flares observed in these systems may be attributed to reconnection (Favata et al. 2005). These processes will act in addition to the effect of the (possibly evolving) dynamo and surface flows.

Recently, we have successfully acquired Zeeman-Doppler images of two of these very young stars that are still accreting from their disks. One of these, BP Tau is only $0.7 \mathrm{M}_{\odot}$ and is believed to be fully convective, while the other, V2129 Oph at $1.4 \mathrm{M}_{\odot}$ is believed to have already developed a radiative core (Donati et al. 2007, 2008; Jardine et al. 2008). As shown in Fig. 5, BP Tau displays a strong (1.2kG) dipolar component to its magnetic field. In contrast, as shown in Fig. 6, the dominant field component in V2129 Oph is the $1.2 \mathrm{kG}$ octupole component. The relatively stronger dipole component of BP Tau's field may allow it to carve out a larger inner hole in its disk, relative to the co-rotation radius (Gregory et al. 2008). 


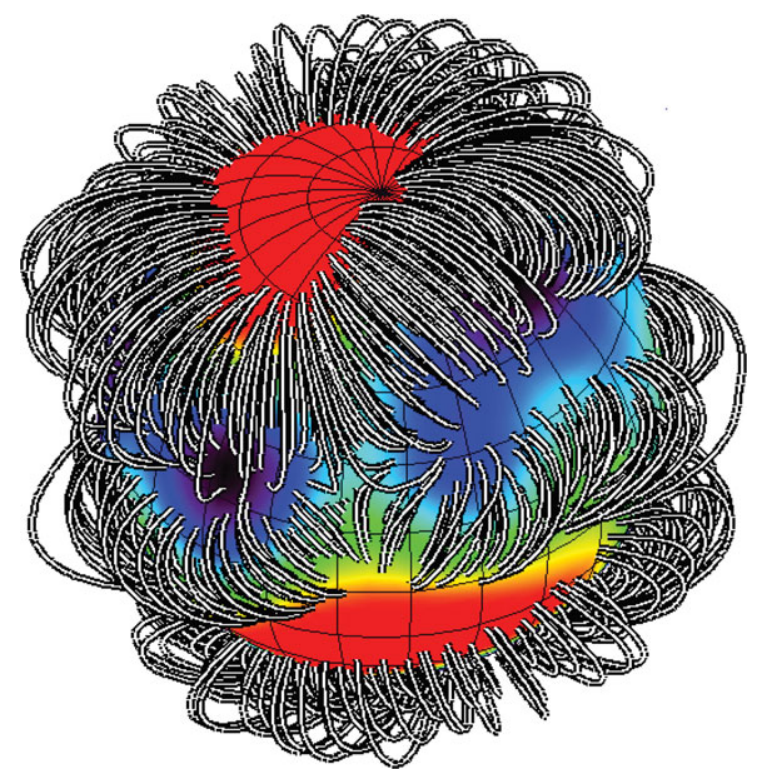

Figure 6. Closed field lines structure extrapolated from Zeeman-Doppler image of V2129 Oph.

\section{Prominences in young stars}

In young stars that are still accreting it would be impossible to detect prominences, even if they were present, since the $\mathrm{H}_{\alpha}$ line is so strongly affected by the accretion process that variations due to prominences could not be disentangled from those due to accretion. However, in stars that have only recently lost their disks, it is possible and indeed in one such example, TWA6, at least one prominence has been detected (Skelly et al. 2008). This is a very interesting example as the star appears to be at the boundary in its evolution between a fully convective state and the development of a radiative core. This star has a heavily-spotted surface with spots extending all the way to the rotation pole. The one prominence detected survived for at least 3 days and was situated at a radius of $4 R_{\star}-$ consistent with the maximum value of $4.8 \mathrm{R}_{\star}$ that would be predicted by the Jardine \& van Balegooijen (2006) theory.

\section{Conclusions}

It is clear from solar observations of prominences that they delineate the structure of the magnetic field, but inferring that structure from observations of prominences is not a simple (or even possible) task. This problem is even more challenging in the case of stellar prominences where their large distance from the stellar rotation axis presents a challenge to models of their confinement by the star's magnetic field. The new Zeeman-Doppler maps of stellar surface magnetic fields, however, show that stellar magnetic fields may be very different in stars of different mass and hence internal structure. In particular, the presence of a convectively stable core - and hence of a shear layer or tachocline separating this from the convective outer region - seems to lead to a complex, high-order field. Stars that are fully convective seem to show a much simpler structure. At present, we only have observations of prominences on mature stars with radiative cores. The low mass stars that are fully-convective are too faint to allow the detection of the transient $\mathrm{H}_{\alpha}$ absorption features that are the signature of prominences. The one example we have 
of prominences forming in a star that is at the boundary between a fully-convective state and the formation of a radiative core is the young star TWA6 which displays a complex field. In a star with a simple dipolar field we might expect prominences to form a torus in the equatorial plane of the star - hence producing no rotational modulation. The detection of prominences in a low mass star would be an interesting test of the field structures detected by Zeeman-Doppler methods, with potentially profound implications for dynamo theories.

\section{References}

Altschuler, M. D. \& Newkirk, Jr., G. 1969, Solar Phys., 9, 131

Barnes, J., Collier Cameron, A., James, D. J., \& Donati, J.-F. 2000, MNRAS, 314, 162

Braithwaite, J. \& Nordlund, A. 2006, A\&A A, 450, 1077

Braithwaite, J. \& Spruit, H. C. 2004, Nature, 431, 819

Browning, M. 2008, ApJ, in press

Brun, A. S., Browning, M. K., \& Toomre, J. 2005, ApJ, 629, 461

Byrne, P., Eibe, M., \& Rolleston, W. 1996, A\&A, 311, 651

Cattaneoe, F. 1999, ApJ, 515, L39

Chabrier, G. \& Küker, M. 2006, $A \& A$, 446, 1027

Charbonneau, P. \& MacGregor, K. B. 2001, ApJ, 559, 1094

Collier Cameron, A. \& Campbell, C. G. 1993, A\&A, 274, 309

Collier Cameron, A. \& Robinson, R. D. 1989a, MNRAS, 238, 657

Collier Cameron, A. \& Robinson, R. D. 1989b, MNRAS, 236, 57

Collier Cameron, A. \& Woods, J. A. 1992, MNRAS, 258, 360

Dobler, W., Stix, M., \& Brandenburg, A. 2006, ApJ, 638, 336

Donati, J.-F. 2001, LNP Vol. 573: Astrotomography, Indirect Imaging Methods in Observational Astronomy, 573, 207

Donati, J.-F. \& Collier Cameron, A. 1997, MNRAS, 291, 1

Donati, J.-F., Collier Cameron, A., Hussain, G., \& Semel, M. 1999, MNRAS, 302, 437

Donati, J.-F., Forveille, T., Cameron, A. C., et al. 2006a, Science, 311, 633

Donati, J.-F., Howarth, I. D., Jardine, M. M., et al. 2006b, MNRAS, 370, 629

Donati, J.-F., Jardine, M. M., Gregory, S. G., et al. 2007, MNRAS, 380, 1297

Donati, J.-F., Jardine, M. M., Gregory, S. G., et al. 2008, MNRAS, 386, 1234

Donati, J.-F., Mengel, M., Carter, B., Cameron, A., \& Wichmann, R. 2000, MNRAS, 316, 699

Dunstone, N. J., Hussain, G. A. J., Cameron, A. C., et al. 2008a, MNRAS, 387, 1525

Dunstone, N. J., Hussain, G. A. J., Collier Cameron, A., et al. 2008b, MNRAS, 387, 481

Durney, B. R., De Young, D. S., \& Roxburgh, I. W. 1993, Solar Phys., 145, 207

Eibe, M. T. 1998, A\& A, 337, 757

Favata, F., Flaccomio, E., Reale, F., et al. 2005, ApJS, 160, 469

Gregory, S. G., Jardine, M., Cameron, A. C., \& Donati, J.-F. 2006a, MNRAS, 373, 827

Gregory, S. G., Jardine, M., Simpson, I., \& Donati, J.-F. 2006b, MNRAS, 371, 999

Gregory, S. G., Matt, S. P., Donati, J.-F., \& Jardine, M. 2008, MNRAS, in press

Hussain, G. A. J., van Ballegooijen, A. A., Jardine, M., \& Collier Cameron, A. 2002, ApJ, 575, 1078

Jardine, M., Barnes, J., Donati, J.-F., \& Collier Cameron, A. 1999, MNRAS, 305, L35

Jardine, M., Collier Cameron, A., \& Donati, J.-F. 2002a, MNRAS, 333, 339

Jardine, M., Collier Cameron, A., Donati, J.-F., Gregory, S. G., \& Wood, K. 2006, MNRAS, 367,917

Jardine, M., Collier Cameron, A., Donati, J.-F., \& Pointer, G. 2001, MNRAS, 324, 201

Jardine, M., Gregory, S. G., \& Donati, J.-F. 2008, $M N R A S$, in press

Jardine, M. \& van Ballegooijen, A. A. 2005, MNRAS, 361, 1173

Jardine, M., Wood, K., Collier Cameron, A., Donati, J.-F., \& Mackay, D. H. 2002b, MNRAS, 336,1364

Jeffries, R. 1993, MNRAS, 262, 369 
Königl, A. 1991, ApJ, 370, L39

Küker, M. \& Rüdiger, G. 1997, A\&SA, 328, 253

Küker, M. \& Rüdiger, G. 1999, in: ASP Conf. Ser. 178: Workshop on stellar dynamos, Vol. 178, $87-96$

Long, M., M., R. M., \& Lovelace, R. V. E. 2007, MNRAS, 374, 436

Lynden-Bell, D. \& Boily, C. 1994, MNRAS, 267, 146

MacDonald, J. \& Mullan, D. J. 2004, MNRAS, 348, 702

Maeder, A. \& Meynet, G. 2005, A\&SA, 440, 1041

Matt, S. \& Pudritz, R. E. 2005, ApJ, 632, L135

McIvor, T., Jardine, M., Cameron, A. C., Wood, K., \& Donati, J.-F. 2003, MNRAS, 345, 601

Moss, D. 2001, in: S. G. Mathys \& D. Wickramasinghe (eds.), ASP Conference Series, Vol. 248, Magnetic fields across the Hertzsprung-Russell diagram, (San Francisco), 305

Mullan, D. J. \& MacDonald, J. 2005, MNRAS, 356, 1139

Rekowski, B. V. \& Brandenburg, A. 2004, A\& $A$, 420, 17

Shu, F., Najita, J., Ostriker, E., et al. 1994, ApJ, 429, 781

Skelly, M. B., Unruh, Y. C., Cameron, A. C., et al. 2008, MNRAS, 385, 708

Spruit, H. 2002, A\& A, 381, 923

Strassmeier, K. 1996, in: K. G. Strassmeier \& J. L. Linsky, (eds.), IAU Symposium 176: Stellar Surface Structure, (Kluwer), 289-298

Tout, C. A. \& Pringle, J. E. 1995, MNRAS, 272, 528

Townsend, R. H. D. \& Owoki, S. P. 2005, MNRAS, 357, 251

\section{Discussion}

DASso: Do you see some relationship between the existence of a convection zone in stars and prominences?

JARDINE: We have only observed prominences in stars that have a convective zone. High mass stars which have a radiative interior do show transient H-alpha absorption features. These are interpreted as a "wind compressed disk" — though they show many features in common with prominences.

SPANGLER: There is a well-established empirical relation for the decrease in X-ray luminosity and coronal activity with time for solar-type stars ("the Sun in time"). Are these data useful as constraints on your magnetic field models?

JARDINE: This decrease is largely due to the change in rotation rate as solar-type stars spin down due to the action of a hot, magnetically-channeled wind. We can only observe prominences on rapidly-rotating stars, so it is difficult to follow this whole evolution.

Gopalswamy: 1. Do you observe eruptive prominences? 2. Are there prominences in flare stars and what happens to the prominences during flares?

JARDINE: 1. Yes, on Speedy Mic, but it isn't published yet. 2. Yes, there are stellar "Active Prominences" that are associated with flares - but we don't know if they are ejected or simply heated.

GIRISH: I believe that the maximum height of observation of a stellar prominence will depend on the latitude of its origin and on the phase of the stellar activity cycles. Have you looked in the long term observations available for solar prominences to verify this idea? 
JARDINE: AB Dor has been observed for over 10 years and there is no evidence for a cyclic change in the magnetic structure, although there is a variation in optical brightness. The nature of magnetic cycles on other stars is as yet an open question.

VRŠNAK: Why our Sun does not have these huge prominences?

JARDINE: It rotates too slowly! Stellar prominences appear to form at or beyond the Keplerian co-rotation radius, which for the Sun is located at $40 \mathrm{R}_{S}$. 